\title{
FACTORES DE RIESGO CARDIOVASCULAR EN POBLACIÓN INDÍGENA Y MESTIZA, EN CHIAPAS
}

\author{
CARDIOVASCULAR RISK FACTORS IN THE INDIGENOUS AND MIXED-RACE POPULATIONS IN CHIAPAS. \\ Cruz-Serrano Nely Isabel ${ }^{1}$, Briones-Aranda Alfredo ${ }^{2}$, Bezares-Sarmiento Vidalma del Rosario ${ }^{1}$, Toledo- Meza \\ María Dolores ${ }^{1}$, León-González Juan Marcos ${ }^{1}$.
}

1 Universidad de Ciencias y Artes de Chiapas. 2 Universidad Autónoma de Chiapas. México.

\begin{abstract}
RESUMEN
Introducción: Las enfermedades cardiovasculares y la incidencia de sus factores de riesgo es alta, con tendencia a incrementar. Objetivo: Describir la prevalencia de riesgo cardiovascular en dos poblaciones: indígena y mestiza en los altos de Chiapas, México. Material y Método: Estudio descriptivo, transversal, muestra obtenida por conveniencia del Hospital de las Culturas en población sin enfermedades cardiovasculares conocida y con diferentes factores de riesgo, se aplicó encuesta prediseñada para evaluar la prevalencia de factores de riesgo modificables. Se realizaron pruebas bioquímicas, evaluación del índice de masa corporal. El análisis estadístico se realizó con nivel de significancia estadística $p<0.05$. Resultados: Participaron 123 adultos ( $66 \%$ de origen indígena, $34 \%$ mestizos). Edad promedio 44 años, analfabetismo $50 \%$, mayor prevalencia de sedentarismo y tabaquismo en mestizos, $73 \%$ de los indígenas y el $50 \%$ de los mestizos se clasificaron con riesgo cardiovascular bajo, el moderado (21\%) y el alto $(29 \%)$ fue mayor en los mestizos. La obesidad está se relacionada con el grupo étnico. Conclusiones: El riesgo cardiovascular es dependiente del grupo étnico; la aculturación, analfabetismo y dialecto representan factores socioculturales que incrementan en población indígena. Las estrategias, la alfabetización en salud y la creación de programas multi e interdisciplinarios en salud, podrían impactar positivamente sobre la disminución del riesgo cardiovascular en estas poblaciones.
\end{abstract}

Palabras Clave: Prevalencia, factores de riesgo, enfermedad cardiovascular, indígenas, mestizos.

\section{ABSTRACT}

Introduction: Cardiovascular diseases and the incidence of their risk factors is high, with a tendency to increase. Objective: To describe the prevalence of cardiovascular risk in two populations: indigenous and mestizo in Los Altos of Chiapas, Mexico. Material and method: Descriptive, cross-sectional study, sample obtained by convenience of the Hospital de las Culturas in a population without known cardiovascular diseases and with different risk factors, a pre-designed survey was applied to evaluate the prevalence of modifiable risk factors. Biochemical tests, evaluation of the body mass index were carried out. Statistical analysis was performed with a statistical significance level $p<0.05$. Results: 123 adults participated (66\% of indigenous origin, $34 \%$ mestizo). Average age 44 years, illiteracy $50 \%$, higher prevalence of sedentary lifestyle and smoking in mestizos, $73 \%$ of indigenous people and $50 \%$ of mestizos were classified with low, moderate (21\%) and high (29\%) cardiovascular risk it was greater in the mestizos. Obesity is related to ethnicity. Conclusion: Cardiovascular risk is dependent on ethnic group; acculturation, illiteracy and dialect represent sociocultural factors that increase the indigenous population. The strategies, health literacy and the creation of multi and interdisciplinary health programs could have a positive impact on the reduction of cardiovascular risk in these populations.

Key words: Prevalence, risk factors, cardiovascular disease, indigenous, mixed race.

Correspondencia: Vidalma del Rosario Bezares-Sarmiento.vidalma.bezares@unicach.mx

Recibido: 25 de julio 2021, aceptado: 20 de septiembre 2021

(C)Autor2021

(c) (i)

DOI: https://doi.org/10.29105/respyn20.4-4

Citation: Cruz-Serrano N.I., Briones-Aranda A., Bezares-Sarmiento V.R., Toledo- Meza M.D., León-

González J.M. (2021) Factores de riesgo cardiovascular en población indígena y mestiza, en Chiapas.

Revista Salud Pública y Nutrición, 20 (4), 31-46. 


\section{Artículo Original}

\section{Introducción}

Entre los principales problemas que enfrenta actualmente la humanidad, se encuentran las enfermedades crónicas no transmisibles (ECNT), que presentan tasas altas tasas de morbilidad, sobre todo problemas cardiovasculares, los cuales están asociados a factores de riesgo modificables y no modificables. Los factores de riesgo cardiovasculares (FRCV), pueden ser estimados por métodos cualitativos y se clasifican en leve, moderado y alto, mientras que los cuantitativos indica la probabilidad de sufrir enfermedades cardiovasculares (ECV) en un tiempo determinado, esto se calcula mediante tablas de riesgo cardiovascular (Ortega, 2021). Estas enfermedades han incrementado ante el cambio de las costumbres y estilos de vida, es bien sabido que la práctica de actividad física (AF), tiene un rol importante en la prevención de enfermedades crónicas como la diabetes mellitus, además de retardar la aparición de otros factores de riesgo cardio metabólicos, que mejoran la calidad de vida y el bienestar psicológico de las personas que padecen estas enfermedades (Paing, Kirk, Collier, Kubiak, y Chastin, 2018). Sin embargo, con los siglos, las ocupaciones manuales y la $\mathrm{AF}$ han disminuido, a favor de profesiones y oficios de mayor acción intelectual y por ende mayor sedentarismo. Actualmente el uso de vehículos de transporte favorece la inactividad física, sumado a la falta de tiempo y la carencia de hábitos por realizar ejercicio (Rivera, Cedillo, Pérez, Flores y Aguilar, 2018). Medina, Jáuregui, Campos y Barquera (2018) reportaron en la ENSANUT-MC, que más del $80 \%$ de los niños y $35 \%$ de los adolescentes en México no cumple con las recomendaciones de actividad física, y que la tendencia no mejoró de 2012 a 2016; por otra parte la ENSANUT, 2018-19, puntualizó que las mujeres adultas a nivel nacional permanecen sentadas 3 horas 30 minutos al día, distinguiéndose que las mujeres de 20 a 29 años de edad es el grupo de personas con mayor tiempo de descanso (4 horas 25 minutos), comparado con los otros grupos de edad; mientras que en el nivel rural las horas que permanecen sentadas es de 3 horas 10 minutos (Medina, Jáuregui, Ríos y Barquera, 2020).

Rivera et al. (2018), señalan que además de otros determinantes como los hábitos de alimentación inadecuados, la premura para conseguir y preparar los alimentos, han tendido a favorecer el apego al consumo de alimentos procesados con elevada densidad calórica e inciden en conjunto en la distribución, frecuencia y magnitud de estas enfermedades.

Los cambios en la cultura alimentaria en México, junto al avance tecnológico han traído como consecuencia, mayor riesgo para desarrollar enfermedades crónicas, entre ellas las ECV, incrementando las prevalencias de morbimortalidad a nivel global (Gómez y Velázquez, 2019).

En Chiapas, de forma similar al resto del país, los padecimientos crónicos constituyen el principal reto en salud, lo que llama a fortalecer la capacidad de respuesta del sector salud ante estas condiciones. Al mismo tiempo, la presencia de estilos de vida no saludables, denotados por la importante prevalencia de consumo de alcohol (Chiapas: $10.8 \%$ población mayor de 20 años) y tabaco (Chiapas: 6.6\% población mayor de 20 años), exacerba el reto de los padecimientos crónicos y llama a fortalecer el abordaje preventivo (INEGI, 2014; INEGI-INSPSSA, 2019).

Por otra parte, la prevalencia mundial de las enfermedades crónicas no trasmisibles (ECNT), como la HTA, DM, la Obesidad y las Dislipidemias, va en ascenso, ya que de acuerdo con datos de la ENSANUT, 2018-19, la prevalencia de DM en México fue de $10.3 \%$, siendo mayor en las mujeres $(11.4 \%)$ que en los hombres $(9.1 \%)$, para hipertensión arterial de $18.4 \%$, mayor en las mujeres $(20.9 \%)$ que en los varones $(15.3 \%)$, e hipercolesterolemia estuvieron en $30.4 \%$, con más prevalencia en población de 40 a 59 años (Rojas, Mendoza y Laguna, 2020). Además, los cambios en el estilo de vida sedentario o con muy baja AF están favoreciendo el incremento en la prevalencia de estos FRCV, en donde, participan e interaccionan factores genéticos y la influencia innegable del medio ambiente. En esta misma línea, el tabaquismo, el alcoholismo, también son otros factores que favorecen la prevalencia de dichas enfermedades y sus complicaciones y todo esto se consideraban exclusivas de los pobladores de grandes urbes en los países desarrollados. Actualmente, estas enfermedades también están presentes entre la población indígena o rural. Ciertas etnias tienen mayor susceptibilidad para desarrollar ECV que otras, especialmente aquellas que emigran a lugares donde su forma de vida, accediendo, entre otras 


\section{Artículo Original}

cosas, a los alimentos industrializados y a espacios laborales que no demandan extenuante actividad física y como consecuencia propician el incremento exponencial de estas patologías (Page, 2014). Brocklebank et al. (2015), en revisión sistemática, identificaron la coincidencia en 28 estudios la asociación entre sedentarismo y FRCV, con marcadores de resistencia a la insulina, por otra parte, Leiva et al. (2017), a este respecto hallaron que el incremento se asocia significativamente con la adiposidad y deterioro de la salud cardiovascular, independientemente de factores socio-demográficos, alimenticios, IMC y AF.

La población de México se ha distinguido a lo largo de la historia por su diversidad étnica, quienes viven en condiciones precarias en materia de ingresos, educación, vivienda y servicios; factores que determinan desigualdad social basada en su ubicación dentro de la jerarquía de poder, prestigio y acceso a los recursos, y que influyen de manera determinante en la salud, y se manifiesta al analizar el proceso de transición epidemiológica en México; en donde la prevalencia de enfermedades crónico degenerativas, aunado a los de déficit nutricios han aumentado en los últimos años. Hecho ocurrido por muchos factores y que varía según las características genéticas de la población, los cambios en los hábitos alimentarios y las costumbres o hábitos relacionados con la AF de los individuos. (Daviglus et al., 2012). Con base al estudio internacional de Framingham, ideal para realizar el estudio epidemiológico, se ha tomado en cuenta los criterios de este, en donde se puede identificar los principales FRCV, relacionados con el medio ambiente y las características propias del paciente como: tabaquismo, HTA, hipercolesterolemia, antecedentes familiares de ECV, obesidad, DM, sedentarismo, hipertrigliceridemia, cHDL, edad, género y condiciones psicosociales (Rodríguez et al., 2011). Kershaw, Greenlund, Stamler, Shay, y Daviglus (2012) determinaron las características individuales que contribuyen durante el seguimiento a la aparición posterior de la ECV. Por otra parte, Hurley et al. (2012), demostró la existencia que en otros grupos raciales/étnicos puede verse afectada por diferencias en la prevalencia y la importancia relativa de estos factores de riesgo, por no ser identificados y que siguen estando mal definidos en los grupos raciales. de igual manera, Dinwiddie, Zambrana, y Garza (2014), en sus resultados de los FRCV, señalaron las diferencias en la prevalencia por la influencia de factores como la educación, lugar de nacimiento y el género; observando que a mayor grado de estudio se asoció con mayores probabilidades de HTA en hombres y en mujeres mayor probabilidad de padecer DM. Guzmán y Roselló (2011), indicaron en su estudio que las variables sociodemográficas (sexo, edad, ocupación y educación), antropométricas (peso, talla e IMC), factores de riesgo como tabaquismo, DM, HTA, obesidad y datos bioquímicos (CT, cHDL, cLDL y glicemia) tienen una relación importante.

La prevalencia de DM se ha modificado en los últimos años, según el reporte de la ENSANUT, 2018-19, hace el análisis de los informes de los años 2012 y 2018 (9.2\% vs $10.3 \%$ respectivamente) La prevalencia entre los hombres y mujeres de 60 a 69 años, presenta una ligera variación entre ambos reportes, hombres $(8.6 \%$ vs $9.1 \%)$, mujeres $(9.7 \%$ vs $11.4 \%$ ), observándose que fue ligeramente mayor en mujeres (Rojas et al., 2020).

Con respecto a la hipertensión arterial se observaron cambios del reporte de 2012 y 2018 , en donde hubo un incremento en la prevalencia a partir de los 40 años con un incremento relativo de $208.7 \%$ en mujeres $(22.9 \%$ vs $47.8 \%)$ y de $208.3 \%$ en hombres ( $16.9 \%$ vs $35.2 \%$ ), en general después de los 20 años, esta prevalencia de hipertensión es reportada como de $16.6 \%$ (2012), y $18.4 \%$ para 2018 . A nivel nacional, la prevalencia de HTA por diagnóstico médico previo en el estado de Chiapas se ubicó debajo de la media nacional que es de $46.8 \%$, disminuyendo ésta para población rural (21.9\%), (Campos et al., 2020).

El reporte de los resultados de CT elevado fue mayor en la población de 40 a 59 años (34.7\%) y en la de 60 años o más $(30.4 \%)$. Se observaron resultados diferentes de CT elevado por sexo en el grupo de 40 a 59 años (34\% en hombres y $42.1 \%$ en mujeres) y en el de 60 años o más $(33.4 \%$ en hombres y $42.1 \%$ en mujeres) (Rojas et al., 2020).

La prevalencia de sobrepeso y obesidad (IMC $\geq 25$ $\mathrm{kg} / \mathrm{m}^{2}$ ) en hombres fue de $73.0 \%$, y en mujeres, de $76.8 \%$. Al comparar la prevalencia de obesidad fue en hombres $(30.5 \%)$ que en mujeres $(40.2 \%)$, siendo $9.7 \%$ más alta en mujeres que en varones (Medina, Jáuregui, Ríos, Barquera, 2020). 
Alcocer, Lozada, Fanghänel, Sánchez, y Campos (2011), muestran la estimación del RCV, que se ha convertido en piedra angular de las guías clínicas de prevención primaria para el tratamiento de la dislipidemia. Las tablas de Framingham y de Evaluación Sistemática de Riesgo Coronario (SCORE), han sido útiles para determinar la probabilidad de sufrir un evento cardiovascular; en México se han utilizado las tablas de bajo riesgo SCORE, ya que, de acuerdo con la OMS, epidemiológicamente le corresponde la del grupo B (baja mortalidad infantil y en adultos), más parecida a la de países europeos con bajo riesgo.

Tablas de riesgo de Framingham. Utiliza un método de puntuación con base a las siguientes variables: edad (35 - 74 años), sexo, cHDL, colesterol total, presión arterial sistólica, tabaquismo (si/no), diabetes (si/no), e hipertrofia ventricular izquierda (HVI) (si/no), con ello se puede calcular el riesgo coronario a los 10 años que incluye: angina de pecho estable, infarto al miocardio y muerte coronaria (Sans, Fitzgerald, Royo, Conroy, Graham, 2007).

Modelo SCORE. En Europa, múltiples Sociedades Cardiovasculares; propusieron un nuevo algoritmo denominado Systematic Coronary Risk Evaluation (SCORE), que fue presentado en 2003. El proyecto reunió un pool de bases de datos de estudios de cohortes de 12 países europeos (Finlandia, Rusia, Noruega, Gran Bretaña, Escocia, Dinamarca, Suecia, Bélgica, Alemania, Italia, Francia y España), principalmente poblacionales, con 88.080 mujeres y 117.098 hombres, 2,7 millones de personas-año de seguimiento, y 7.934 muertes cardiovasculares, de las que 5.652 fueron muertes coronarias (Yusuf et al., 2004). A partir de este pool de datos, se derivó una regla de predicción del riesgo de ECV mortal en 10 años, en el que la edad es usada como una medida del tiempo de exposición. Los FR incluidos para cada sexo en función de la edad son: consumo de tabaco, PAS y CT o cociente CT/HDLc. SCORE permite estimar la probabilidad de muerte cardiovascular, tanto por enfermedad coronaria como por enfermedad cerebrovascular para individuos de entre 40 y 65 años, en los pacientes menores de 40 años, se calculó el riesgo proyectando su edad a 40 años. Para los pacientes mayores de 65 años, se estimó el riesgo como si su edad fuera 65 años (Sans et al., 2007; Saab et al., 2015).
Dada la variabilidad geográfica del RCV en Europa, se desarrollaron dos modelos SCORE, para países de alto o bajo riesgo. De los dos modelos desarrollados, uno basado en el CT y otro en la razón CT / C - HDL, en el estudio se utilizó el basado en el CT. La novedad más importante de la función de riesgo SCORE comparada con la de Framingham es que estima el riesgo mortal de todas las manifestaciones aterotrombóticas cardiovasculares. La lógica de este cambio es que los mismos FR están asociados con diversas enfermedades y que las personas con alto riesgo de muerte CV también lo están de episodios no mortales (Sans et al., 2007). El estudio realizado en población adulta de Huancayo-Perú, asoció los factores sociales, fisiológicos y el RCV, según el Framingham Risk Score, el riesgo de padecer un evento CV a 10 años fue bajo en el $88 \%$ de los casos (Mejía et al., 2016).

En el proceso de adaptación de las guías de prevención, se aconsejó calibrar los modelos SCORE al nivel de riesgo de cada país SCORE, ha sido desarrollado para definir el estilo de vida, FR y terapéuticas para la prevención de las ECV. (Banegas et al., 2011).

La importancia de la realización de este estudio fue mostrar la información disponible de las desigualdades existentes en la salud de la población indígena y no indígena de Chiapas y evaluar el papel que tiene el grupo étnico en la explicación de tales desigualdades. Asimismo, se analizó la relación entre las condiciones de vida de la población indígena y su estado de salud, lo cual posibilitará indagar más a los FR tradicionales y justificará la realización de investigaciones con el propósito de estimar el RCV y cualquier actitud, recomendación o intervención que haya demostrado la capacidad para mejorar la calidad de vida de las personas o de disminuir su morbimortalidad. Es así como el objetivo de este trabajo fue describir la prevalencia de los FRCV en dos poblaciones, una indígena y otra mestiza que residen en la zona de los altos de Chiapas en espera del cambio en la tendencia actual para contribuir a implementar programas de salud pública tendientes a reducirlos.

\section{Material y Método}

Estudio descriptivo, transversal, para determinar la prevalencia de FRCV en dos poblaciones sin ECV conocida, con diferentes componentes étnicos y 
sociodemográficos para estimar el riesgo cardiovascular, realizado en el hospital de las culturas, ubicado en la ciudad de San Cristóbal de la Casas, en el estado de Chiapas, México, nosocomio en donde se utiliza medicina tradicional, de concentración de pacientes indígenas (Instituto de Salud del Estado de Chiapas, 2013). Se integró una muestra no probabilística a conveniencia del investigador. La población de estudio estuvo conformada por 123 pacientes atendidos en la consulta externa del hospital, distribuidos en 81 de origen indígena y 42 mestizos. Se incluyeron pacientes adultos de ambos sexos, entre 18 a 60 años, indígenas y mestizos, obesos o no obesos, que cursaran o no con dislipidemias, DM, presión arterial (HTA), quienes autorizaron su participación mediante carta de consentimiento informado personal o a través de un traductor o familiar en los casos de no hablar el idioma español. Excluyendo pacientes que presentaran complicaciones y/o gravedad de su padecimiento en el momento del estudio.

Para la recolección de datos, se llevó a cabo una anamnesis realizada en la primera visita, por medio de la aplicación de cuestionario, diseñada en cinco apartados: datos de identificación, antecedentes socio-familiares, antecedentes sobre estilo de vida (actividad física, consumo de alcohol, tabaco), además de realizarse exámenes bioquímicos, para ello, a cada paciente se le extrajeron muestras de sangre venosa tras ayuno de 8 horas, por la mañana, en tubo de vacío sin anticoagulante, para la determinación de: Glucosa, Urea, Creatinina, Ácido úrico, Colesterol total, Colesterol ligado a lipoproteínas de alta densidad. (HDL), lipoproteínas de baja densidad (LDL) y lipoproteínas de muy baja densidad (VLDL), Triglicéridos. Las muestras de sangre se centrifugaron a $3500 \times$ g durante 3 minutos en una centrífuga SOLBAT a 4o C, y el plasma se eliminó y analizó inmediatamente después de la recolección. Para la determinación cuantitativa de glucosa, colesterol total y triglicéridos en suero, Se utilizó el método enzimático colorimétrico (GLUCOSA PAP SL, ELITECH, Francia). Para determinar el colesterol HDL se utilizó el método de precipitación combinado con un método enzimático colorimétrico (CHOD-PAP, RANDOX LAB LTD); la medición de triglicéridos se llevó a cabo mediante método enzimático, hidrólisis con lipasas (GPOPPAP, RANDOX, Reino Unido). El colesterol LDL se calculó mediante el método de Friedewald. fórmula: LDL $(\mathrm{mmol} / \mathrm{L})=$ colesterol total $-(\mathrm{TG} / 5)-\mathrm{HDL}$, precisa para muestras con valores inferiores a $400 \mathrm{mg}$ / dl. Todas las mediciones fueron realizadas por un espectrofotómetro. RA-50 (Sistema de química clínica, Bayer, Alemania). El principio del método para determinación de urea es la hidrolisis de esta, catalizada por medio de la ureasa presente en la muestra en amoniaco $\left(\mathrm{NH}^{3}\right)$ y anhídrido carbónico $\left(\mathrm{CO}^{2}\right)$, el amoniaco se incorpora al $\alpha$-cetoglutarato por acción del glutamato deshidrogenasa (GLDH) con oxidación paralela de NADH a NAD+, la disminución de la concentración del NAD+ es proporcional a la concentración de urea en la muestra. El ácido úrico es oxidado por la uricasa a alantoína y peróxido de hidrógeno $\left(2 \mathrm{H}^{2} \mathrm{O}^{2}\right)$, que en presencia enzimática se convierte en un compuesto rosáceo la quinonaimina y la intensidad roja es proporcional a la concentración de ácido úrico. Para todas estas determinaciones se utilizó un espectrofotómetro Spinlab o analizador para lecturas a 510, 340 y $520 \mathrm{~nm}$ respectivamente. La medición de la creatinina estuvo basada en la reacción con el picrato de sodio descrita por Jaffe formando un complejo rojizo que de acuerdo con la intensidad es proporcional a la concentración de creatinina, todas se expresan en $\mathrm{mg} / \mathrm{dL}$. El peso corporal obtenido en $\mathrm{Kg}$ fue medido en una balanza con precisión de 0.1 $\mathrm{kg}$, la talla en $\mathrm{cm}$ con una precisión de $1 \mathrm{~cm}$. Se calculó el índice de Quetelet o índice de masa corporal $(\mathrm{IMC})$ mediante la fórmula: $\mathrm{IMC}=$ peso corporal $(\mathrm{Kg}) /$ Talla $(\mathrm{m})^{2}$. Para determinar obesidad se utilizó el indicador de IMC (Suverza y Haua, 2010; Luna, Coello, León, Pascacio, y Bezares, 2014), se siguió las técnicas en apego a los criterios de la Norma Oficial Mexicana NOM-008-SSA32017 (SSA, 2018), para el tratamiento integral del sobrepeso y la obesidad, indicado en el apartado 15. Apéndice a informativo. A.1 Puntos de corte de los indicadores antropométricos. Para la medida de la presión arterial clínica (PAC) se siguieron las recomendaciones de la Sociedad de Hipertensión Británica: El paciente sentado, con el brazo izquierdo descubierto en ligera flexión y el antebrazo apoyado sobre una superficie lisa a la altura del corazón, recomendándole no hubiera ingerido cafeína ni haber fumado en los 30 minutos previos a la medición. Durante 5 minutos previos a la medida de la PA, permaneció en reposo en un ambiente tranquilo y de temperatura confortable. Se realizaron 3 medidas a intervalos de 3 minutos de reposo. Se obtuvo la 
media de la segunda y tercera medida. La presión se expresa en milímetros de mercurio ( $\mathrm{mmHg}$ ), se siguieron las recomendaciones y clasificación de la hipertensión arterial según la Norma Oficial Mexicana NOM-030-SSA2-2009 para la prevención, tratamiento y control de la hipertensión arterial (SSA, 2010a). Para la presencia de dislipidemia se utilizaron los valores recomendados por la NOM 037-SSA2-2002 para la prevención, tratamiento y control de las dislipidemias (SSA, 2012). El diagnóstico de DM se establece según los criterios de la NOM-015-SSA2-2010 para la prevención, tratamiento y control de la DM (SSA, 2010b).

La estratificación del RCV de forma clínica, se tomó como base los 4 niveles o categorías del riesgo propuestas por el NCEP-ATP III y revisadas en el 2004 (riesgo bajo, moderado y riesgo alto). Para la estimación del RCV se utilizaron las tablas de predicción del riesgo de la Organización Mundial de la Salud (OMS, 2008), que indican el riesgo de padecer un episodio cardiovascular grave, mortal o no, en un periodo de 10 años según la edad, el sexo, la presión arterial, el consumo de tabaco, el colesterol total en sangre, con presencia o ausencia de DM; los niveles de riego estimado están interpretados como: Riesgo de episodio cardiovascular a 10 años: $<10 \%$ bajo, 10 a $<20 \%$ moderado, $>20$ a $<30 \%$ alto (Álvarez, 2001).

En el análisis estadístico de la información se utilizaron las variables que eran las que se relacionaban con los objetivos del estudio, tres dependientes (glucemia, presión arterial y niveles de colesterol), viendo la significancia mediante la Chi cuadrado $\left(\mathrm{x}^{2}\right)$, con intervalo de confianza del $95 \%$, y significancia de $\mathrm{p}<0.05^{*}$. El análisis descriptivo consistió en el cálculo de media \pm y desviación estándar en las variables cuantitativas continúas. Las variables cualitativas categóricas se calcularon mediante el porcentaje de hombres y mujeres, se utilizaron medidas descriptivas de resumen para la edad y el IMC general y por sexo. En cuanto a las medidas antropométricas se dividió a la población de estudio tomando en consideración el IMC: sobrepeso (de 25 a 29.9) y obesidad (de 30 o mayor). Se procedió a dicotomizar las variables independientes numéricas con la finalidad de poder realizar el análisis en las tablas $2 \times 2$ mediante, el programa estadístico empleado fue el EPI INFO, para Windows versión 6 .

\section{Resultados}

De las 123 personas participantes, procedentes de los Altos de Chiapas, 58 fueron hombres (47.3\%) y 65 mujeres $(52.7 \%)$, con promedio de edad $44 \pm 16.8$ años, indígena $42.6 \pm 18$ años vs mestizo $45.23 \pm$ 15.7 años (Tabla 1$)$.

Tabla 1. Media de la edad por grupo étnico y sexo

\begin{tabular}{lcccc}
\hline & $\begin{array}{c}\text { Indígena } \\
\mathrm{n}=81\end{array}$ & \multicolumn{2}{l}{$\begin{array}{l}\mathrm{o} \\
\mathrm{n}=42\end{array}$} \\
& Media & DS & Media & DS \\
\hline Sexo & 47.0 & 17.9 & 50.0 & 15.8 \\
\hline Hombres & 38.0 & 16.2 & 41.0 & 13.9 \\
Mujeres & 42.6 & 18.0 & 45.2 & 15.7 \\
Total & & & &
\end{tabular}

La distribución de la población total por grupo étnico fue, 81 personas indígenas $(66 \%$ de la población total), y 42 personas mestizas ( $34 \%$ de la población total). En la población indígena (40\%) eran analfabeta, característico del grupo étnico $\left(\mathrm{X} 2=12.01 ; \mathrm{p}<0.05^{*}\right), 60 \%$ cursaba con primaria, en comparación con la población mestiza $(10 \%)$, en donde la mayoría eran profesionistas, mostrando diferencia estadística importante (X2=13,25; p $\left.<0.05^{*}\right)$. En lo laboral, el grupo étnico al que pertenecen los participantes está asociado con el tipo de ocupación que realizan $\left(X 2=38.89 ; \mathrm{p}<0.05^{*}\right)$, en el grupo indígena predominó las labores del hogar $(51 \%)$, y del campo (32\%). Finalmente, se observa que tanto la actividad física $\left(\mathrm{X} 2=11.38 ; \mathrm{p}<0.05^{*}\right)$ como el hábito tabáquico $\left(\mathrm{X} 2=5.21 ; \mathrm{p}<0.05^{*}\right)$ están asociados de manera significativa con el grupo étnico, mostrando que los mestizos presentan mayor sedentarismo y tabaquismo. En lo que se refiere al consumo de alcohol, se observó que la población mestiza aparentemente presenta mayor frecuencia de consumo de alcohol (40\%), sin embargo, no refirió diferencia significativa (Tabla 2). 
Tabla 2. Distribución de la población según variables sociodemográficas

\begin{tabular}{|c|c|c|c|c|c|c|}
\hline \multirow[b]{2}{*}{ Variable sociodemográfica } & \multicolumn{2}{|c|}{$\begin{array}{c}\text { Indígena } \\
\mathrm{n}=81\end{array}$} & \multicolumn{2}{|c|}{$\begin{array}{c}\text { Mestizo } \\
\mathrm{n}=42\end{array}$} & \multicolumn{2}{|c|}{ TOTAL } \\
\hline & $\mathrm{n}$ & $\%$ & $\mathrm{n}$ & $\%$ & $\mathrm{n}$ & $\%$ \\
\hline \multicolumn{7}{|l|}{ Sexo } \\
\hline Hombres & 37 & 46.0 & 21 & 50.0 & 58 & 47.2 \\
\hline Mujeres & 44 & 54.0 & 21 & 50.0 & 65 & 52.8 \\
\hline \multicolumn{7}{|l|}{ Alfabetismo* } \\
\hline Analfabeta & 32 & 40.0 & 4 & 10.0 & 36 & 29.3 \\
\hline Alfabeta & 49 & 60.0 & 38 & 90.0 & 87 & 70.7 \\
\hline \multicolumn{7}{|l|}{ Escolaridad* } \\
\hline Primaria & 33 & 41.0 & 13 & 31.0 & 46 & 52.9 \\
\hline Secundaria & 9 & 11.0 & 9 & 21.0 & 18 & 20.7 \\
\hline Preparatoria & 6 & 7.0 & 8 & 19.0 & 14 & 16.1 \\
\hline Licenciatura & 1 & 1.0 & 8 & 19.0 & 9 & 10.3 \\
\hline \multicolumn{7}{|l|}{ Ocupación* } \\
\hline Oficios del hogar & 41 & 51.0 & 14 & 33.0 & 55 & 44.7 \\
\hline Campesino & 26 & 32.0 & 1 & 2.0 & 27 & 22.0 \\
\hline Comerciante & 4 & 5.0 & 4 & 10.0 & 8 & 6.5 \\
\hline Albañil & 4 & 5.0 & 1 & 2.0 & 5 & 4.1 \\
\hline Profesionistas & 6 & 7 & 22 & 52.0 & 28 & 22.8 \\
\hline \multicolumn{7}{|l|}{ Ejercicio* } \\
\hline Activo & 70 & 86 & 25 & 60.0 & 95 & 77.2 \\
\hline Sedentario & 11 & 14 & 17 & 40.0 & 28 & 22.8 \\
\hline \multicolumn{7}{|l|}{ Tabaquismo** } \\
\hline Fumador & 14 & 17 & 15 & 36.0 & 29 & 23.6 \\
\hline No fumador & 67 & 83 & 27 & 64.0 & 94 & 76.4 \\
\hline \multicolumn{7}{|l|}{ Alcoholismo } \\
\hline Bebedor & 25 & 31 & 17 & 40 & 42 & 34.1 \\
\hline No bebedor & 56 & 69 & 25 & 60 & 81 & 65.9 \\
\hline
\end{tabular}

De La e manera general la mayor proporción fue representada por tener un riesgo bajo (65\%), seguido de un riesgo alto (18\%) y finalmente en su menor proporción un riesgo moderado (17\%) (Figura 1).

Figura 1. Prevalencia de riesgo cardiovascular en la población.

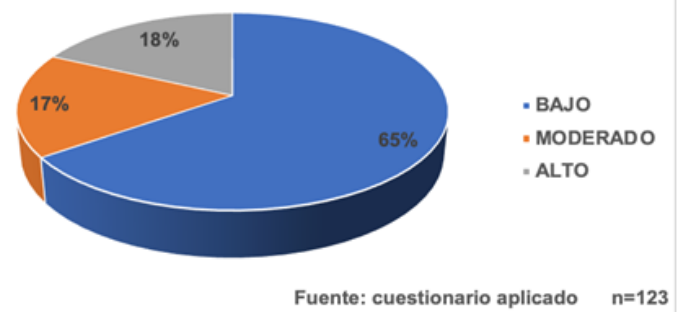

Tanto el RCV bajo como alto, muestran una dependencia significativa con el grupo étnico. En ambos casos también se observa una mayor prevalencia de RCV bajo en indígenas y RCV alto en los mestizos (Tabla 3).

\begin{tabular}{lcccccc}
\multicolumn{7}{c}{ Tabla 3. Estimación de riesgo cardiovascular en indígenas y } \\
mestizos
\end{tabular}

Fuente: encuesta

${ }^{*} \mathrm{p}=.01$

$* * p<.05$

Finalmente, cuando se analiza las posibles asociaciones entre la presencia de los diversos FRCV en relación con el grupo étnico al que pertenecen los participantes, se observa que la presencia de obesidad está relacionada significativamente con el grupo étnico, con mayor prevalencia en el grupo de los mestizos en un $31 \%$ (Tabla 4). 
Tabla 4. Distribución del factor de riesgo cardiovacular presente en la población total

\begin{tabular}{|c|c|c|c|c|c|c|}
\hline \multirow{2}{*}{$\begin{array}{l}\text { Factores } \\
\text { de riesgo } \\
\text { cardiovascular }\end{array}$} & \multicolumn{2}{|c|}{$\begin{array}{l}\text { Indígenas } \\
n=81\end{array}$} & \multicolumn{2}{|c|}{$\begin{array}{c}\text { Mestizos } \\
n=42\end{array}$} & \multicolumn{2}{|c|}{ TOTAL } \\
\hline & $\mathrm{n}$ & $\%$ & $\mathrm{n}$ & $\%$ & $\mathrm{n}$ & $\%$ \\
\hline \multicolumn{7}{|l|}{ Sobrepeso } \\
\hline SI & 34 & 42.0 & 14 & 33.0 & 48 & 39.0 \\
\hline NO & 47 & 58.0 & 28 & 67.0 & 75 & 61.0 \\
\hline \multicolumn{7}{|l|}{ Obesidad } \\
\hline SI & 9 & 11.0 & 13 & 31.0 & 22 & 17.9 \\
\hline NO & 72 & 89.0 & 29 & 69.0 & 101 & 82.1 \\
\hline \multicolumn{7}{|c|}{ Hipertensión Arterial } \\
\hline SI & 21 & 26.0 & 13 & 31.0 & 34 & 27.6 \\
\hline NO & 60 & 74.0 & 29 & 69.0 & 89 & 72.4 \\
\hline \multicolumn{7}{|c|}{ Diabetes Mellitus } \\
\hline SI & 32 & 40.0 & 22 & 52.0 & 54 & 43.9 \\
\hline NO & 49 & 60.0 & 20 & 48.0 & 69 & 56.1 \\
\hline \multicolumn{7}{|c|}{ Dislipidemias agrupadas } \\
\hline SI & 73 & 90.0 & 34 & 81.0 & 107 & 87.0 \\
\hline NO & 8 & 10.0 & 8 & 19.0 & 16 & 13.0 \\
\hline \multicolumn{7}{|c|}{ Hipertrigliceridemia } \\
\hline SI & 48 & 59.0 & 22 & 52.0 & 70 & 56.9 \\
\hline NO & 33 & 41.0 & 20 & 48.0 & 53 & 43.1 \\
\hline \multicolumn{7}{|c|}{ Hipercolesterolemia } \\
\hline SI & 9 & 11.0 & 4 & 10.0 & 13 & 10.6 \\
\hline NO & 72 & 89.0 & 38 & 90.0 & 110 & 89.4 \\
\hline \multicolumn{7}{|c|}{ Hipoalfalipoproteinemia } \\
\hline SI & 59 & 73.0 & 29 & 69.0 & 88 & 71.5 \\
\hline NO & 22 & 27.0 & 13 & 31.0 & 35 & 28.5 \\
\hline \multicolumn{7}{|l|}{ Colesterol LDL } \\
\hline SI & 6 & 7.0 & 3 & 7.0 & 9 & 7.3 \\
\hline NO & 75 & 93.0 & 39 & 93.0 & 114 & 92.7 \\
\hline \multicolumn{7}{|l|}{ Colesterol VLDL } \\
\hline SI & 37 & 46.0 & 17 & 40.0 & 54 & 43.9 \\
\hline NO & 44 & 54.0 & 25 & 60.0 & 69 & 56.1 \\
\hline
\end{tabular}

\section{Discusión}

Los cambios económicos, sociales, políticos y culturales han modificado las tendencias nutricionales y epidemiológicas en México. La adopción de un estilo de vida occidental, han modificado la composición de la dieta y de la actividad física, dado lugar al aumento en la prevalencia de los FRCV y metabólicos como las dislipidemias, HTA, tabaquismo, DM, obesidad (Ferreira et al., 2017).

Otro problema que incide en la salud de la población es la escolaridad, en general, la población indígena se caracteriza por ser analfabeta (518 361), en estados como Chiapas y Oaxaca, es mayor que las del resto del país (Rojas et al., 2015); por otra parte, aunado al alto índice de marginación y pobreza que presenta la población indígena. La población indígena de los Altos de Chiapas presenta cambios culturales, al adoptar el modo de vida, partiendo que ya empiezan a hablar el idioma español, lo que denota la proximidad de la convivencia en las zonas urbanas y con población no indígenas, lo cual ha generado su trasformación de esta población, cambiando su forma de vida tradicional, como lo es el caso de atención de la salud-enfermedad, haciendo uso de la medicina tradicional, lo cual se mantiene pero ha manifestado cambios importantes, por su acercamiento a área urbana, que los ha llevado en consecuencia a la exposición a factores de riesgo, característicos del proceso de urbanización, que ha ido modificando las de costumbres tradicionales, con la consecuente adopción de estilo de vida occidental (Adam et al., 2014; Ferreira et al., 2017; López y Teodoro, 2006; OPS/OMS 2012), como pudo observarse en esta investigación.

Aun cuando en los resultados se halló predominio de riesgo bajo a presentar un evento cardiovascular, si es destacable mencionar, que la presencia de enfermedades crónicas no transmisibles, es coincidente con el estudio de Cruz, Tuñón, Villaseñor, Álvarez y Nigh (2013), en donde se encontró que dentro de los FRCV identificados se obtuvo a 70 pacientes $(57 \%)$ con alteraciones de peso, con IMC alto; 48 (39\%) con sobrepeso y 22 (18\%) con obesidad, siendo más prevalente en mujeres. La alta prevalencia de sobrepeso encontrado en la población indígena coincide con el incremento en el sobrepeso y obesidad reportado previamente en las comunidades indígenas de los altos de Chiapas; lo que podría estar relacionado con el incremento en el consumo de bebidas azucaradas y gasificadas en estos habitantes (Cruz et al., 2013). Por otra parte, el estudio realizado por Paramio, Letrán, Requesen y Hernández (2021), calcularon el RCV en 376 personas, encontrándose que el $63.30 \%$ tuvieron RCV-global bajo, y que a medida que aumenta la edad el RCV global también tiende a aumentar, con mayor prevalencia en hombres; también observaron que el $24.74 \%$ presentaron cifras de hipertensión arterial sistólica mayor o igual a $140 \mathrm{mmHg}$, y el $29.26 \%$ registraron cifras de colesterol por encima de 


\section{Artículo Original}

$6 \mathrm{mmol} / \mathrm{L}$, siendo estos factores de riesgo posibles de modificar.

De igual manera, es interesante observar el incremento en la prevalencia del tabaquismo, alcoholismo, situación coincidente en otras poblaciones indígenas latinoamericanas (Cardona et al., 2012; Oliveira et al., 2014). La prevalencia de tabaquismo en este estudio fue menor a la reportada en el estudio del Carmen de $26,9 \%$ y mayor que el estudio ENFREC II de $22,7 \%$. Lo mismo sucede con el alcohol, 42 hombres (34\%) consume alcohol. La prevalencia del consumo de alcohol en este estudio fue superior al reportado en Chile $(13,2 \%)$ y a la reportada en el estudio del Carmen que reportó 18,7 $\%$ de la población, siendo inferior al informado $(37,4$ $\%$ ) en Colombia (Catano et al., 2015).

Los factores de riesgo identificados en el estudio, detectada como problemática grave, por el hecho de que la mayoría de las personas que los presentan, no están conscientes de su presencia. Otra de las condiciones encontradas en este estudio, fue que la mayoría de los participantes indígenas se dedicaba predominantemente a labores del hogar y el campo, semejante que en otras regiones como en Colombia, el hombre le compete la caza y pesca y la recolección de frutas, la construcción de la vivienda, las labores referentes a la preparación de la siembra, la construcción de los medios de transporte. En Brasil existen varios grupos, los tikuna se dedican a la pesca, los maku a la colecta de miel (Rubio, 2014) lo que sugiere mayor actividad física, en contraste con la población mestiza que se dedican a labores más sedentarias; encontrándose entre los mestizos mayor grado de RCV; por otra parte, el incremento progresivo del RCV observado en el grupo indígena, posiblemente está influenciado por el proceso de aculturación, por la transición de las poblaciones indígenas al sedentarismo y a la adopción de hábitos alimentarios urbanos, así como la incorporación de alcohol y tabaco (Aluli et al., 2009; Ferreira et al., 2017). Por otra parte, un caso similar estudiado en indígenas de la Amazonia Brasileña, indican que cambios socioculturales, económicos y ambientales son factores de riesgo cardiovascular importantes en los cambios encontrados en esa población (Sombra et al., 2021).

De acuerdo a los resultados de este estudio, la población indígena se observó ser mayormente activos que la mestiza, ya que esta muestra mayor prevalencia de sedentarismo, lo cual puede atribuirse a la ocupación que desempeñan, por ser en su mayoría profesionistas o amas de casa, comparado con las investigaciones realizadas (Araque, Ceballos, Fernández, y Montalvo, 2017; Cardona, 2012), refieren posibles factores sobre la escasa actividad física, entre los que se destacan: la falta de tiempo, de voluntad y de energía, con la consecuente adopción de conductas poco saludables, como el ver más tiempo televisión o consultar el internet. Según la OPS, en algunos países ha disminuido la actividad física de la población, lo que se ha asociado con procesos de urbanización y cambios en los estilos de vida. En la población indígena de la presente investigación, se observó una proporción importante que se dedica al hogar o al comercio, lo cual les limita la realización de ejercicio físico; estudios como los de Catano (2015), señala que, las principales barreras para la actividad física, es la falta de tiempo, de voluntad y de energía, lo que muestra similitud con los Emberá-Chamí, e implica la adopción de conductas poco saludables y la pérdida del arraigo cultural en esta comunidad indígena.

Con respecto a la HTA, es el FR con mayor impacto en el aumento de morbilidad y la mortalidad por $\mathrm{ECV}$, independientemente del efecto de otros factores. Este FR se encontró en 34 (28\%) pacientes, 21 eran indígenas $(26 \%)$ y 13 mestizos $(31 \%)$, estas cifras fueron similares a las estimaciones realizadas para América Latina, donde se reporta un 35\%, en Chile un $36.7 \%$, en la Encuesta Nacional de Salud y Nutrición de México se halló un 43.2\%. Sin embargo, algunos estudios en Colombia y España han reportado prevalencias del $74.5 \%$, en Argentina del 76\% (INEGI, 2015).

De acuerdo a los resultados del presente estudio, el consumo de alcohol y tabaco en población indígena fue importante, aunque fue menor que en la población mestiza, no obstante es mayor el porcentaje, si se compara con el estudio realizado por López et al. (2020) en población indígena con DM sobre la prevalencia de tabaquismo y alcoholismo de manera general fue de $7.4 \%$ y de tabaco de $3.1 \%$, siendo mayor en hombres que en mujeres, en donde posiblemente haya la influencia del entorno sociocultural; entre los indígenas comunidades tzotziles y tzeltales de los altos de Chiapas, el aguardiente consumido es el pox o posh, considerada 


\section{Artículo Original}

como una bebida ritual y tradicional, símbolo de fraternidad entre las comunidades (Reyes, 2009).

En las alteraciones en las concentraciones de lípidos encontrados en este estudio, se observó la prevalencia mayor entre la población indígena $(87 \%)$, principalmente de hipoalfalipoproteinemia, estudios han dado a conocer el efecto de la omega 3 en la disminución del proceso de hígado graso y de regular receptores nucleares que controla el metabolismo del colesterol, además de otras vías glucolíticas. La segunda dislipidemia más frecuente son los TG altos, combinada con HDL bajos y con VLDL alto, y la tercera dislipidemia más frecuente es la VLDL alta combinada con TG altos y/o con HDL baja. Las cifras de glucosa altas se presentaron en 54 (44\%), 28 (23\%) hombres y 26 (21\%) mujeres (Castellanos y Rodríguez, 2015).

El estudio costarricense de Hernández (2016), muestra que un 77,6 \% de la población tienen dos o más factores de riesgo para ECV, datos muy semejantes a los de este trabajo y a los descritos por otros investigadores colombianos. De igual manera, los resultados del presente estudio son similares a los reportados en la literatura científica internacional en el estudio de MONICA; sin embargo, se sabe que los factores modificables como el sedentarismo, los hábitos de alimentación, el consumo de alcohol y tabaco, pueden atenderse antes de los 40 años, ya que después de esa etapa de vida el desarrollo de la DM es inminente (Vázquez et al., 2019).

\section{Conclusiones}

La OMS ubica la ECV dentro de las primeras cinco causas de mortalidad en adultos de ambos sexos; proyecta que para el 2030 dicha enfermedad representará la causa de más de 8 millones de muertes a partir de los 30 años. En la actualidad las sociedades de cardiología de todo el mundo se han interesado en los FRC y su repercusión en la expectativa de vida. Eso motivó nuestra inquietud y la realización del presente estudio.

El nivel de RVC está asociado significativamente con el tipo de grupo étnico, en donde los mestizos presentaron mayor prevalencia de RCV alto. Dentro de los principales factores asociados, la obesidad fue el único factor asociado significativamente al grupo étnico. Aunque la población indígena fue la que ocupó el segundo lugar en la prevalencia de RCV, es preocupante la tendencia del incremento de peso presente en esta población, posiblemente por diversos factores socioculturales entre los que se destacan el proceso de aculturación, la barrera del idioma, el analfabetismo.

El nivel educativo cumple un rol importante en el desarrollo de ECV e influye en el RCV. Por un lado, la población con mejor nivel educativo tiende a tener mayor riesgo coronario, además de estar relacionado con el nivel socioeconómico, considerándose que, a más recursos económicos, mayor RCV.

Se ha observado que los indígenas de los altos de Chiapas por la cercanía que tienen con la capital del estado viajan frecuentemente a ella por múltiples motivos principalmente por dedicarse al comercio de su mercancía y por lo tanto algunos permanecen mucho tiempo allí e incluso algunos ya viven en la capital y la transición del estilo de vida al sedentarismo y a hábitos alimenticios urbanos, así como la incorporación de alcohol y tabaco, determina un aumento en el promedio de la presión arterial, e incremento a más edad, característica de las sociedades occidentales. El estilo de vida sedentario o la baja AF- junto con el hábito de fumar y el consumo de alcohol- potencian el desarrollo de factores de riesgo hacia el sobrepeso y obesidad que se consideran buenos predictores de FRCV, hábitos que tienden a persistir toda la vida, y están asociados con dislipidemias, HTA, aterosclerosis; dando lugar a mayor riesgo de desarrollar cardiopatías coronarias.

El cálculo del riesgo $\mathrm{CV}$ total sigue siendo herramienta fundamental en la prevención de enfermedad cardiovascular, dando lugar a que las personas con mayor riesgo de un episodio de ECV sean las más beneficiadas de las medidas preventivas. El 35\% de la población total de estudio presento un riesgo cardiovascular moderado y alto, con predomino en la población mestiza en comparación con el grupo de los indígenas.

En Chiapas, de forma similar al resto del país, los padecimientos crónicos constituyen el principal reto en el sector salud, lo que llama a fortalecer la capacidad de respuesta de este sector ante estas condiciones. Al mismo tiempo, la presencia de estilos de vida no saludables exacerba el reto de los padecimientos crónicos, y llama a fortalecer el abordaje preventivo. Así pues, se concluye que la 


\section{Artículo Original}

estimación del riesgo cardiovascular es la forma más razonable y costo-efectivo para determinar las prioridades de prevención cardiovascular en personas asintomáticas.

\section{Fortalezas.}

Este estudio aporta información de un grupo poblacional, correspondiente a la mayor prevalencia de los FRC en la zona de los altos de Chiapas, en estrecha relación con la obesidad, sedentarismo, y marcadores de desventaja socioeconómica, por consiguiente, los estudios de prevalencia de los FRC siguen estando justificados para generar hipótesis y orientar políticas sanitarias costo-efectiva de determinar las prioridades en prevención cardiovascular en personas asintomáticas, plantear mejores campañas educativas y fortalecer los programas de control en la comunidad, particularmente la de fortalecer la etno-educación, la interculturalidad en salud y la recuperación de la identidad cultural y que, además, permita asignar los recursos en función de las necesidades, entendiendo como tal es el riesgo de presentar una ECV. Poder determinar el RCV de un individuo se ha convertido en una herramienta esencial en la prevención de la ECV.

Los estudios sobre FRC en indígenas resultan necesarios, puesto que la mayoría de las investigaciones sobre la enfermedad y otros factores se han desarrollado en poblaciones diferentes. Los resultados del estudio pueden extrapolarse a grupos con características similares a las descritas en este manuscrito; por lo cual es de gran relevancia para investigaciones posteriores.

\section{Limitaciones.}

La principal limitación de nuestro estudio es que se trata de un estudio transversal en un sector de la población específico, que es incapaz de establecer relaciones causales. Los resultados, por lo tanto, son representativos de un colectivo concreto en Chiapas, por lo que, se debe tener el cuidado que cuando se realicen estudios similares al referido, cubran las mismas características en cuanto a la población estudiada, para evitar sesgos en la obtención de resultados y estos sean diferentes.

Por otro lado, es difícil comparar las prevalencias de los FRC con otros estudios epidemiológicos, debido a las diferencias en la metodología empleada, los grupos de edad estudiados, la estandarización de la población, ocupaciones (en el caso de los que analizan población laboral), los factores estudiados y la escala de riesgo considerados. Algunos determinantes históricos culturales del grupo retrasaron la recolección de la información en algunas comunidades.

\section{Recomendaciones.}

A la luz del análisis del comportamiento epidemiológico de la patología cardiovascular se hace evidente una génesis multifactorial, con ello se fundamenta la importancia de la detección y estudio de los FRC, ya que al sospechar o diagnosticar dichas enfermedades, es posible ofertar oportunamente el manejo y con ello un control clínico-metabólico, además de evitar o retrasar las complicaciones inherentes a dichas enfermedades. De ahí que la propuesta del presente trabajo sea además de la identificación por el profesional médico de los FRC en el sujeto aparentemente sano, conocer precisamente cuánto sabe y por ende reconoce dicho sujeto respecto a los denominados FRC.

La importancia de fomentar la interculturalidad en salud constituye una estrategia para mejorar el acceso y la calidad de la atención, para proporcionar a los pueblos los medios necesarios para promoverla y tener mayor control de sus vidas. La meta es prevenir futuros eventos cardiovasculares.

Teniendo en cuenta que las ECV constituyen una de las principales causas de mortalidad y morbilidad en el país, es necesario que sea de gran interés el desarrollo de modelos de predicción del riesgo de padecer este tipo de enfermedades. Recomendar el uso de los diagramas de predicción del riesgo basados en el estudio SCORE (del inglés "Systematic Coronary Risk Evaluation", 'evaluación sistemática del riesgo coronario') es un adelanto muy importante para identificar a las personas con un elevado RCV $\mathrm{y}$, en especial, para motivar a los pacientes a modificar la intensidad de las conductas de reducción de riesgo en forma individualizada, para mejorar la adherencia en terapias de reducción de riesgo. Además, la educación en salud, es una de las medidas a emplear en el fomento de una alimentación sana, un peso deseable y un estilo de vida activo que incluya un programa mediante campañas preventivas, asesoramiento alimentario, sugerencias 
respecto a la $\mathrm{AF}$ o en su momento con tratamientos terapéuticos.

En resumen, la gran ventaja del abordaje de la calificación del riesgo es que proporciona medios racionales para tomar decisiones acerca de la intervención de manera orientada, lo que permite un mejor uso de los recursos para reducir el RCV. Es preciso realizar más investigaciones que validen los diagramas actuales de predicción del riesgo subregionales para poblaciones concretas en los niveles nacional y local, y para confirmar que el uso de métodos de estratificación del riesgo en países de ingresos bajos y medianos da lugar a beneficios tanto para los pacientes como para el sistema de atención de salud.

\section{Bibliografía}

Adam, A. L., Lambrick, D. M., Faulkner, J. A., Fryer, S., Tarrant, M. A., Podevigne, M., Williams, M. A. y Stoner, L. (2014). Modifiable Cardiovascular Disease Risk Factors among Indigenous Populations. Hindawi Publishing Corporation Advances in Preventive Medicine: 1-13. DOI: $10.1155 / 2014 / 547018$. Disponible en: https://eprints.soton.ac.uk/382253/1/Modifiable_ cardiovascular_disease_risk_factors_among_ind igenous_populations.pdf

Alcocer L, Lozada O, Fanghänel G, Sánchez L, Campos E. (2011). Estratificación del riesgo cardiovascular global. Comparación de los métodos Framingham y SCORE en población mexicana del estudio PRIT. Cirugía y Cirujanos. 79: $168-174$.

Álvarez, A. (2001). Las tablas de riesgo cardiovascular: Una revisión crítica. Medifam, 11 (3), 122-139. Disponible en: https://scielo.isciii.es/pdf/medif/v11n3/revision. pdf

Aluli, N. E., Jones, K. L. Reyes, P. W., Brady, S. K., Tsark, J. U., y Howard, B. V. (2009). Diabetes and Cardiovascular Risk Factors in Native Hawaiians. Journal Hawaii Medicine, 68 (7): 152-157.

Araque, A. M., Ceballos, F. A., Fernández, J. J., Montalvo, M. (2017). La comunidad emberá- chamí de Dojura (Chigorodó, Antioquia): tensiones jurídicas y resistencia vital. Boletín de Antropología, (32) 53: 142-157.

Banegas, J. R., Díez-Gañán, L., Bañuelos-Marco, B., González-Enríquez, J., Villar-Álvarez, F., Martín-Moreno, J. M., Jiménez-Ruiz, C. (2011). Smoking-attributable deaths in Spain, 2006. Medicine Clinical, 136, 97-102. DOI: 10.1016/j.medcli.2010.03.039

Brocklebank, L., Falconer, C., Page, A., Perry, R., \& Cooper, A. R. (2015). Accelerometer-measured sedentary time and cardiometabolic biomarkers: A systematic review. Preventive Medice, 76, 92102. doi: 10.1016/j.ypmed.2015.04.013.

Campos, I., Hernández, L., Espinosa, J., Padilla, D., y Barquera, S. (2020). Estado de nutrición y tensión arterial en adultos. Encuesta Nacional de Salud y Nutrición 2018-19: Resultados Nacionales. Cuernavaca, México: Instituto Nacional de Salud Pública.

Cardona, J. A. (2012). Prevalencia de factores de riesgo cardiovascular en indígenas de RiosucioCaldas, 2010-2011. Revista Medicina UPB, 31(2): 113-126. Disponible en: https://revistas.upb.edu.co/index.php/medicina/a rticle/view/1658

Castellanos, L., y Rodríguez, M. (2015). El efecto de omega 3 en la salud humana y consideraciones en la ingesta. Revista Chilena de Nutrición, 42(1), 90-95. DOI: $\quad 10.4067 / \mathrm{S} 0717$ 75182015000100012

Catano, J. U., Duque, J., Naranjo, C. A., Rúa, D. C., Rosique, J., García, A. F., Pizano, N. D. (2015). Prevalencia de factores de riesgo cardiovascular en indígenas embera-chami de Cristiania (Jardín), Antioquia. IATREIA, 28(1): 5-16. Disponible en: http://aprendeenlinea.udea.edu.co/revistas/index. php/iatreia/article/view/18535

Cruz, M., Tuñón, E., Villaseñor, M., Álvarez, G. C., y Nigh, R. B. (2013). Sobrepeso y obesidad: una propuesta de abordaje desde la sociología. Región y sociedad, XXV (57): 165-202. 
Daviglus, M. L., Talavera, G. A., Aviés, M. L. Allison, M., Cai, J., Criqui, M. H...Stamle, J. (2012). Prevalence of Major Cardiovascular Risk Factors and Cardiovascular Diseases among Hispanic/Latino Individuals of Diverse Backgrounds in the United States. Journal of American Medical Association, 308(17), 17751784. DOI: 10.1001/jama.2012.14517.

Dinwiddie, G. Y., Zambrano, R. E., y Garza, M. A. (2014). Explorando los factores de riesgo en las enfermedades cardiovasculares de los Latinos: el papel de la educación, nacimiento y género. American Journal Public Health. 104(9): 17421750. DOI: 10.2105/AJPH.2013.301280

Ferreira, A. A., Souza, Z. A., Goncalves, M. J. F., Santos, J., y Pierin, A. M. G. (2017). Relationship between alcohol drinking and arterial hypertension in indigenous people of the Mura ethnics, Brazil. PLoS One 12(8): e0182352. DOI: 10.1371/journal.pone.0182352. Disponible en: https://pubmed.ncbi.nlm.nih.gov/28777805/

Gómez, Y., y Velázquez E. B. (2019). Salud y cultura alimentaria en México. Revista Digital Universitaria, 20 (1), 1-11.

DOI:

http://doi.org/10.22201/codeic.16076079e.2019. v20n1.a6.

Guzmán, S., y Roselló, M. (2011). Riesgo cardiovascular global en la población adulta del área urbana del Cantón Central de Cartago, Costa Rica. Revista Costarricense de Cardiología, 8 (3), 11-17.

Hernández, W. (2016). Estudio Proyecto SCORE. Documento electrónico: Factores de riesgo de enfermedad cardiovascular en una población obrera industrial de la provincia de Cartago. Revista Costarricense de Salud Pública, 9(16), 55-64

Hurley L., Dickinson M, O Raymond. F. J., P Stein y Havranek E. (2012). Predicción de muerte cardiovascular en las minorías raciales / étnicas utilizando los factores de riesgo de framingham. División de Medicina Interna General, Denver, División de Cardiología. Servicio de Medicina
Familiar de la Universidad de Colorado en Denver.

Instituto de Salud del Estado de Chiapas (ISECH). (2013). Programa Estatal de salud 2013 - 2018. Departamento de Informática y estadística. Hospital de las Culturas. San Cristóbal de las Casas, Chiapas.

Instituto Nacional de Estadística y Geografía (INEGI). (2015). Principales resultados de la encuesta Intercensal 2015. Estados Unidos Mexicanos, México: INEGI, c2015 xi, 72-26p. Disponible en: http://internet.contenidos.inegi.org.mx/contenido s/productos/prod_serv/contenidos/espanol/bvine gi/productos/nueva_estruc/702825078966.pdf

Instituto Nacional de Estadística y Geografía (INEGI). (2014). Perspectiva estadística Chiapas. México: INEGI, c2014. Disponible en: http://internet.contenidos.inegi.org.mx/contenido s/Productos/prod_serv/contenidos/espanol/bvine gi/productos/integracion/estd_perspect/mar_201 4/chis/702825059446.pdf

Instituto Nacional de Estadística y Geografía (INEGI), Instituto Nacional de Salud Pública (ISP) y Secretaría de Salud (SSA). (2019). Encuesta nacional de Salud y Nutrición 2018. Presentación de resultados. México: INEGIINSP-SSA. Disponible en: https:/www.inegi.org.mx/contenidos/programas /ensanut/2018/doc/ensanut_2018_presentacion_r esultados.pdf

Kershaw, N., Greenlund, K. J., Stamler, J., Shay, C. M., y Daviglus, M. L. (2012). Understanding Ethnic and Nativity-Related Differences in Low Cardiovascular Risk Status among MexicanAmericans and Non-Hispanic Whites. Preventic Medicine. 55(6): 597-602. DOI: 10.1016/j.ypmed.2012.09.019

Leiva, A. M., Martínez, M. A., Montero, C. C., Salas, C., Ramírez, R., Díaz, X., Aguilar, N., y Celis, C. (2017). El sedentarismo se asocia a un incremento de factores de riesgo cardiovascular y metabólicos independiente de los niveles de actividad física. Revista Médica Chilena, 145, 
458 - 467. http://dx.doi.org/10.4067/S003498872017000400006

López, J. R., y Teodoro, J. M. (2006). La cosmovisión indígena Tzotzil y Tzental a través de la relación salud-enfermedad en el contexto de la medicina tradicional indígena. Ra Ximhai, 2 (1), 15-26 Disponible en: http://www.revistas.unam.mx/index.php/rxm/arti cle/view/6860/6380

López, L. C., Martínez, Y., Martínez, E., Telumbre, J. Y., Higuera, J. L., y Mendoza, G. (2020). Consumo de alcohol y tabaco en indígenas mexicanos con diabetes mellitus tipo 2. Revista Iberoamericana educación Investigación en enfermería, 10 (4), 35-43.

Luna, M. A., Coello, V. G., León, J. M., Pascacio, M. R., y Bezares, V. R. (2014). Evaluación del estado de nutrición del adulto. En V. R. Bezares, R. M. Cruz, M. Burgos, y M. E. Barrera. (Eds.), Evaluación del estado de nutrición en el ciclo vital humano (pp. 109-122). México: AMMFEN y Mc Graw Hill.

Medina, C., Jáuregui, A., Campos, I., y Barquera S. (2018). Prevalencia y tendencias de actividad física en niños y adolescentes: resultados de ENSANUT 2012 y ENSANUT MC 2016. Salud Pública de México, 60, 263-271.

Medina, C., Jauregui, A., Ríos, V., y Barquera, S. (2020). Encuesta Nacional de Salud y Nutrición 2018-19. Actividad física en niños, adolescentes y adultos. Resultados Nacionales. Cuernavaca, México: Instituto Nacional de Salud Pública.

Mejía, C. R., Espejo, R. P., Zevallos, K. R., Castro, T. A., Vargas, A., y Millan, G. K. (2016). Factores asociados al riesgo cardiovascular según Framingham en taxistas de una empresa de Huancayo, Perú. Revista de la Asociación Española de Especialistas en Medicina del Trabajo, 25 (1), 19-25.

Oliveira, G. F., Oliveira, T. R. R., Ikejiri, A. T., Andraus, M. P., Galvao, T. F., Silva, M. T., y Pereira, M. G. (2014). Prevalence of Hypertension and Associated Factors in an Indigenous Community of Central Brazil: A
Population-Based Study. PLoS ONE, 9(1): 1-6. DOI: 10.1371/journal.pone. 0086278

Organización Panamericana de la Salud (OPS)/Organización Mundial de la Salud (OMS). (2012). La diabetes muestra una tendencia ascendente en las Américas. Washington, D.C. Disponible en: http://www.paho.org/hq/index.php?option=com _content $\&$ view $=$ article $\&$ id $=7453 \% 3$ Adiabetesshows-upward-trend-in-theamericas\&catid $=740 \% 3$ Anews-pressreleases\&Itemid=1926\&lang=es.

Ortega, M. I. (2021). Factores de riesgo cardiovascular. Revista electrónica Portales Médicos, XVI (2), 92-94. Disponible en; https://www.revistaportalesmedicos.com/revista-medica/factoresde-riesgo-cardiovascular/

Paing, A.C., Kirk, A.F., Collier, A., Kubiak, T., y Chastin, F. M. (2018). Are glucose profiles wellcontrolled within the targets recommended by the International Diabetes Federation in type 2 diabetes? a meta-analysis of results from continuous glucose monitoring based studies. Diabetes Research and Clinical Practice, 46, 289-299. DOI: 10.1016/j.diabres.2018.10.010

Page, J. T. (2014). Enfermedades del rezago y emergentes desde las ciencias sociales y la salud pública. México: Instituto de Investigaciones Antropológicas Universidad Nacional Autónoma de México.

Paramio, A., Letrán, Y., Requesen, R. L., y Hernández, M. (2021). Riesgo Cardiovascular Global en el consultorio10 del Policlínico Mártires de Calabazar. Municipio Boyeros. Revista Cubana de cardiología y Cirugía Cardiovascular, 27 (1), 1-7. Disponible en: http://www.revcardiologia.sld.cu/index.php/revc ardiologia/article/view/1008/pd

Reyes, L. (2009). Ingesta de alcohol entre indígenas de Chiapas. Estudio de cuatro casos. LiminaR, 7 (1), 176-189.

Rivera, J. A., Cedillo, L., Pérez, J., Flores, B., y Aguilar, R. I. (2018). Uso de tecnologías, 
sedentarismo y actividad física en estudiantes universitarios. Revista Iberoamericana de Ciencias, 5 (1), 17-23. Disponible en: http://www.reibci.org/publicados/2018/feb/2600 103.pdf

Rodríguez, F., Graciani, A., Guallar, P., León, L. M., Zuluaga, C., López, E., Regidor, E. (2011). Justificación y Métodos del estudio sobre nutrición y riesgo cardiovascular en España (ENRICA). Revista Española de Cardiología, 64(10), 876-882. DOI: 10.1016/j.recesp.2011.05.019

Rojas, M., Rosales, Y., Guerrero, N., Morillo, J., Añez, R. J., Bermúdez, V., y Rojas, J. (2015). Comportamiento epidemiológico de la hipertensión arterial en individuos adultos del municipio San Cristóbal del estado Táchira Venezuela. Revista Latinoamericana de Hipertensión, 10 (2), 25-35. Disponible en: <http://www.redalyc.org/articulo.oa?id=170247 129001>

Rojas, R., Mendoza, L. R., y Laguna, I. (2020). Encuesta Nacional de Salud y Nutrición 2018-19. Programas preventivos, diabetes, hipertensión e hipercolesterolemia. Resultados Nacionales. Cuernavaca, México: Instituto Nacional de Salud Pública.

Rubio, J. C. (2014) Censos y población indígena en México: algunas reflexiones. México: CEPAL.

Saab, K. R., Kendrick, J., Yracheta, J. M., Lanaspa, M. A., Pollard, M., y Johnson, R. J. (2015). New Insights on the Risk for Cardiovascular Disease in African Americans: The Role of Added Sugars. Journal of the American Society of Nephrology, $26 \quad$ (2), 247-257. DOI: 10.1681/ASN.2014040393

Sans, S., Fitzgerald, A.P., Royo, D., Conroy, R., y Grahan, L. (2007). Calibración de la tabla SCORE de riesgo cardiovascular para España. Revista Española de Cardiología, 60 (5), 476485. DOI: 10.1016/S0300-8932(07)75064-9

Secretaría de Salud (SSA). (2010a). Norma Oficial Mexicana NOM-030-SSA2-2009, para la prevención, detección, diagnóstico, tratamiento y control de la hipertensión arterial sistémica. (Diario Oficial de la Federación, publicado el 30/julio/2010). México: Gobierno Federal. Disponible en https://www.cndh.org.mx/DocTR/2016/JUR/A7 0/01/JUR-20170331-NOR21.pdf

Secretaría de Salud (SSA). (2010b). Norma Oficial Mexicana NOM-015-SSA2-2010, para la prevención, tratamiento y el control de la diabetes mellitus (Diario Oficial de la Federación, publicado el 23/noviembre/2010). México: Gobierno Federal. Disponible en http://www.dof.gob.mx/normasOficiales/4215/s alud/salud.htm.

Secretaría de Salud (SSA) (2012). Norma Oficial Mexicana NOM-037-SSA2-2012, para la prevención, tratamiento y control de las dislipidemias (Diario Oficial de la Federación, publicado el 13/septiembre/2012). México: Gobierno Federal. Disponible en http://www.dof.gob.mx/nota_detalle.php?codigo $=5259329 \&$ fecha $=13 / 07 / 2012$.

Secretaría de Salud (SSA) (2018). Norma Oficial Mexicana NOM-008-SSA3-2017, para el tratamiento integral del sobrepeso y la obesidad. (Diario Oficial de la Federación, publicado el 18/mayo/2018). México: Gobierno Federal. Disponible en http://www.comego.org.mx/normatividad/NOR MA\%20Oficial\%20Mexicana\%20NOM-008SSA3-

2017,\%20Para\%20el\%20tratamiento\%20integra $1 \% 20$ de1\%20sobrepeso $\% 20 y \% 201$ \% $\% 20$ obesidad .pdf

Sombra, N. M., Gomes, H. L. M., Sousa, A. M., Almeida, G. S., Souza, Z. A., y Toledo, N. N. (2021). Niveles altos de presión arterial y riesgo cardiovascular entre los indígenas Munduruku. Revista Latino-Americana de Enfermagen, 29 (e3477), 1-15. DOI: 10.1590/15188345.4970.3477

Suverza, A., y Haua, K. (2010). El ABCD de la evaluación del estado de nutrición. México: Mc Graw Hill. 
Vázquez, E., Calderón, Z. G., Ruvalcaba, J. A., Ramírez, E. (2019). Sedentarismo, alimentación, obesidad, consumo de alcohol y tabaco como factores de riesgo para el desarrollo de diabetes tipo 2. Journal of Negative and No Positive Results, 4 (10), 1011-1021. DOI: https://doi.org/10.19230/jonnpr.3068

Vega, A.T., Lozano, J. E., Álamo, R., Lleras, S, Escribano, A., De la Iglesia, P. (2007). Diseño de un estudio poblacional del riesgo cardiovascular en Castilla y León a través de los equipos de atención primaria. Gaceta Sanitaria, 21, 84-7. DOI:10.1016/S0213-9111(07)71976-3

Vega, A. T., Ordaz, A., Lozano, J. E., Álamo, R., Lleras, S., García, P. (2019). Validación del índice SCORE y el SCORE para personas mayores en la cohorte de riesgo de enfermedad cardiovascular en Castilla y León. Hipertensión y Riesgo Cardiovascular, 36 (4), 184 -192. https://doi.org/10.1016/j.hipert.2019.02.002

Yusuf, S., Hawken, S., Ounpuu, S., Dans, T, Avezum, A, Lanas, F., Lisheng, L. (2004). Efecto de los factores de riesgo potencialmente modificables asociados con el infarto de miocardio en 52 países (el Estudio INTERHEART): estudio de casos y controles. Lancet, 364 (9438), 937-952. DOI: 10.1016/S0140-6736(04)17018-9 